\title{
Instrumental validity and intra/inter-rater reliability of a novel low-cost digital pressure algometer
}

\author{
Daniel J Mayorga ${ }^{1}$, Carolina F dos Anjos ${ }^{2}$, Maria C Macedo ${ }^{2}$, Ilha G Fernandes ${ }^{2}$, Esteban A Muñoz ${ }^{3}$, Leonardo \\ Intelangelo $^{4}$, Alexandre Barbosa ${ }^{\text {Corresp. } 2}$ \\ 1 Facultad de Ciencias de la Rehabilitación, Universidad Andrés Bello, Santiago, Santiago, Chile \\ 2 Department of Physical Therapy, Federal University of Juiz de Fora, Governador Valadares, Minas Gerais, Brazil \\ 3 Department of Physical Education, Federal University of Juiz de Fora, Governador Valadares, Minas Gerais, Brazil \\ 4 Department of Physical Therapy, Universidad del Gran Rosario, Rosario, Santa Fe, Argentina \\ Corresponding Author: Alexandre Barbosa \\ Email address: alexandre.barbosa@ufjf.edu.br
}

Background: Pain assessment is a key measure that accompanies treatments in a wide range of clinical settings. A low-cost valid and reliable pressure algometer would allow objective assessment of pressure pain to assist a variety of health professionals. However, the pressure algometer is often expensive, which limits its daily use in both clinical and research settings. Objectives: This study aimed to assess the instrumental validity, and the intra- and inter-rater reliability of an inexpensive digitally adapted pressure algometer. Methods: A single rater applied 60 random compressions on a force platform. The pressure pain thresholds of 20 volunteers were collected twice (three days apart) by two raters. The main outcome measurements were as follows: the maximal peak force (in $\mathrm{kPa}$ ) and the pressure pain threshold (adapted pressure algometer vs. force platform). Cronbach's $\alpha$ test was used to assess internal consistency. The standard error of measurement provided estimates of measurement error, and the measurement bias was estimated with the Bland-Altman method, with lower and upper limits of agreement. Results: No differences were observed when comparing the compression results ( $P=$ 0.51 ). The validity and internal intra-rater consistencies ranged from 0.84 to 0.99 , and the standard error of measurement from 0.005 to $0.04 \mathrm{kPa}$. Very strong $(r=0.73-0.74)$ to near-perfect ( $r=0.99$ ) correlations were found, with a low risk of bias for all measurements. The results demonstrated the validity and intra-rater reliability of the digitally adapted pressure algometer. Inter-rater reliability results were moderate $(r=$ 0.55-0.60; Cronbach' s $\alpha=0.71-0.75)$. Conclusion: The adapted pressure algometer provide valid and reliable measurements of pressure pain threshold. The results support more widespread use of the pressure pain threshold method among clinicians. 


\section{Instrumental validity and intra/inter-rater reliability of a}

2 novel low-cost digital pressure algometer

3

4 Daniel Jerez-Mayorga ${ }^{1}$, Carolina Fernanda dos Anjos $^{2}$, Maria de Cássia Macedo², Ilha

5 Gonçalves Fernandes ${ }^{2}$, Esteban Aedo-Muñoz ${ }^{3}$, Leonardo Intelangelo ${ }^{4}$, Alexandre Carvalho

6 Barbosa $^{2}$

7

Corresponding Author:

Alexandre Carvalho Barbosa ${ }^{2}$

R. Leonardo Cristino, 3400 - São Geraldo, Gov. Valadares, Minas Gerais, CEP 35012000, Brazil Email address: alexwbarbosa@hotmail.com

\section{Abstract}

Background: Pain assessment is a key measure that accompanies treatments in a wide range of clinical settings. A low-cost valid and reliable pressure algometer would allow objective assessment of pressure pain to assist a variety of health professionals. However, the pressure algometer is often expensive, which limits its daily use in both clinical and research settings. Objectives: This study aimed to assess the instrumental validity, and the intra- and inter-rater reliability of an inexpensive digitally adapted pressure algometer.

Methods: A single rater applied 60 random compressions on a force platform. The pressure pain thresholds of 20 volunteers were collected twice (three days apart) by two raters. The main outcome measurements were as follows: the maximal peak force (in $\mathrm{kPa}$ ) and the pressure pain threshold (adapted pressure algometer vs. force platform). Cronbach's $\alpha$ test was used to assess 
31 internal consistency. The standard error of measurement provided estimates of measurement 32 error, and the measurement bias was estimated with the Bland-Altman method, with lower and 33 upper limits of agreement.

34 Results: No differences were observed when comparing the compression results $(\mathrm{P}=0.51)$. The 35 validity and internal intra-rater consistencies ranged from 0.84 to 0.99 , and the standard error of 36

\section{Introduction} measurement from 0.005 to $0.04 \mathrm{kPa}$. Very strong $(\mathrm{r}=0.73-0.74)$ to near-perfect $(\mathrm{r}=0.99)$ correlations were found, with a low risk of bias for all measurements. The results demonstrated the validity and intra-rater reliability of the digitally adapted pressure algometer. Inter-rater reliability results were moderate $(r=0.55-0.60$; Cronbach's $\alpha=0.71-0.75)$.

Conclusion: The adapted pressure algometer provide valid and reliable measurements of pressure pain threshold. The results support more widespread use of the pressure pain threshold method among clinicians.

Pain has been described as a multidimensional construct involving psychological and physical domains with different patterns depending on the emotional state. ${ }^{1}$ These characteristics may impair conclusions and lead to biased clinical reasoning regarding the patterns of group pain due to intra-group and longitudinal variability in subjects' co-morbidities and momentaneous emotional state. Nevertheless, physical assessment is essential to provide objective data to compare the prospective effects of interventions for pain management. ${ }^{2-4}$

1 Pain is mostly assessed by patient self-reports using the visual analog scale. ${ }^{5}$ Self-reported pain intensity is important and reflects physiological and psychological features. However, it can be difficult to interpret because of its subjectivity and overestimation of pain level. ${ }^{6}$ Objective pain assessment is essential to establish a prospective evaluation, compare baseline results to other timeline assessments, or even as a prognostic measure to predict future outcomes. ${ }^{5,7}$ Thus, pressure algometry is a diagnostic aid used to assess some musculoskeletal problems. The pressure pain threshold has been used to aid the diagnosis of pain by providing a quantified force value of tissue tenderness and occurs at the minimum transition point when the applied pressure is sensed as pain. ${ }^{8,9}$ The pressure algometer is an instrument used to assess the pressure pain threshold for both regional and widespread musculoskeletal pain. ${ }^{10}$ This equipment includes a system to convert the force applied through a $1 \mathrm{~cm}^{2}$ pressure application surface to Newtons 
$62\left(\mathrm{~N} / \mathrm{cm}^{2}\right)$ or kilograms of force $\left(\mathrm{kgf} / \mathrm{cm}^{2}\right)$, and a display. The units can be easily converted to 63 kilopascal $(\mathrm{kPa})$, the international metric for pressure $\left(1 \mathrm{~kg} / \mathrm{cm}^{2}=98.066 \mathrm{kPa}\right)$. The pressure 64 algometer enables the rater to semi-objectively quantify the mechanical sensitivity to pain level 65 and the recovery of underlying problems or soreness levels. ${ }^{8,11}$

66 The instrumental validity of commercial pressure algometers has already been assessed in 67 previous studies. Kinser et al. ${ }^{8}$ and Vaughan et al. ${ }^{12}$ manually applied pressure on a force 68 platform to test the reliability and construct validity of pressure algometers. Both studies found 69 high levels of correlation between the force platform and pressure algometers. Other studies have 70 assessed the responsiveness of a pressure algometer to diagnose dysfunctional conditions. Ko et

72 73 74 75 76 77 78 79 al. ${ }^{13}$ assessed the correlation between a modified pressure algometer and a commercial algometer to assess the pressure pain threshold of the epigastric region. Unfortunately, commercially available pressure algometers are expensive and may require specific software for reporting and viewing the results, resulting in more time and training required to assess the pressure pain threshold. The validation of an easy-to-read, low-cost, digitally adaptable pressure algometer would enable widespread quantitative measurements of pressure pain thresholds in clinical practice routine, benefiting early assessment of pain conditions in low-income and developing countries, mainly in primary care. ${ }^{8,14}$ A portable pressure algometer adapted from a hanging scale may be a cost-effective alternative to ensure accurate algometry assessments. The hanging scale is a battery-operated instrument used to weigh objects suspended from an attachment. The equipment uses a load cell, which is a metallic sturdy element, yet elastic enough for a load to deform it. The load cell is attached to a strain gauge, which reads the change in electrical resistance when a pressure or traction load is placed in the load cell. The change in electrical resistance is converted to a digital signal by the strain gauge, and the result appears on a display. ${ }^{15}$ Among other measures, the correlation level between the result of a certain instrument and some external criterion must be confirmed to determine the instrumental validity of an equipment. The criterion has to be a widely accepted measure and considered as the goldstandard method, with the same measurement characteristics of the assessment tool. ${ }^{16,17}$ The purpose of this study was to examine the instrumental validity as well as the intra- and interrater reliability of a low-cost pressure algometer adapted from a hanging scale. Validity was assessed by comparing differences in the measurements of a series of random peak forces applied on a laboratory-grade force platform. Force platforms measure vertical ground reaction 
93 forces in response to compressions applied on the surface. They are considered as the gold94 standard devices for ground reaction forces owing to their high measurement precision. ${ }^{14,18}$ The 95 hypothesis is that a low-cost adapted pressure algometer is valid and reliable to be considered an 96 acceptable method to assess the pressure pain threshold.

97

98

99

100

101

102

103

104 105

106

107

108

109

110

111

112

113

114

115

116

117

118

119

120

121

122 123

\section{Materials \& Methods}

\section{Equipment}

All data were collected at the facilities of the Clinic-School of Physical Therapy, Federal University of Juiz de Fora, in May 2019. The low-cost adapted pressure algometer (MED.DOR Ltd., Brazil; maximum compression $=50 \mathrm{kgf}$, precision $=0.1 \mathrm{kgf}, 3$-digit display) had a $5-\mathrm{cm}$ screw attached to the distal extremity. A $1-\mathrm{cm}^{2}$ round rubber application surface was attached to follow the standardization for pressure algometry (Figure 1). The low-cost adapted pressure algometer calibration is checked by placing a known weight $(1 \mathrm{~kg})$ on the application surface. The maximal tolerated difference between the weight and the value on the display is $0.1 \mathrm{kgf}$. The adapted algometer used in the present study was brand new, and the calibration was checked twice before any measurement.

A two-axis force platform $(37 \mathrm{~cm} \times 37 \mathrm{~cm}$; PASCO, Pasport PS-2142, Roseville, USA) collected data using five force beams (sample rate $=1,000 \mathrm{~Hz}$ ). Four beams in the corner were used to measure the vertical force (range: $-1,100 \mathrm{~N}$ to $+4,400 \mathrm{~N}$ ) and a $5^{\text {th }}$ beam measured the force in a parallel axis (range: $-1,100 \mathrm{~N}$ to $+1,100 \mathrm{~N})$. The recorded trials were converted to $\mathrm{kPa}\left(1 \mathrm{~kg} / \mathrm{cm}^{2}\right.$ $=98.066 \mathrm{kPa})$.

\section{INSERT FIGURE 1 HERE}

\section{Procedures}

\section{Instrumental validity}

An independent rater performed 60 random 3-s pressure trials using the adapted pressure algometer on the force platform with an interval of $3 \mathrm{~s}$. Data were collected and stored using the PASCO Capstone Software (Version 1.13.4, PASCO Scientific, 2019), and the low-cost adapted pressure algometer display readings were recorded using an off-board USB synchronized camera. 
125

126

127

128

129

130

131

132

133

134

135

136

137

138

139

140

141

142

143

144

145

146

147

148

149

150

151

152

153

154

\section{Intra- and inter-rater reliability}

Before the assessment of any participant, two independent raters performed a 4-day training protocol. The protocol consisted of applying constant-progressive pressure with a low-cost adapted pressure algometer on a laboratory-grade load cell (Miotec ${ }^{\mathrm{TM}}$ Biomedical Equipment, Porto Alegre, RS, Brazil; maximum tension-compression $=200 \mathrm{kgf}$, precision $=0.1 \mathrm{kgf}$, maximum error $=$ measurement $=0.33 \%$ ) with Miotec $^{\mathrm{TM}}$ software for visual feedback (MioTrainerTM, Biomedical Equipment, Porto Alegre, RS, Brazil) for two nonconsecutive days (3 nonconsecutive hours per day). The conversion from analog to digital signals was performed by an A/D board (Miotec ${ }^{\mathrm{TM}}$, Biomedical Equipment) with a 16-bit resolution input range, a sampling frequency of $2 \mathrm{kHz}$, a common rejection module greater than $100 \mathrm{~dB}$, a signal-noise ratio less than $03 \mu \mathrm{V}$, root mean square, and impedance of $109 \Omega$. All data were recorded and processed using the Miotec Suite ${ }^{\mathrm{TM}}$ software (Miotec ${ }^{\mathrm{TM}}$ Biomedical Equipment, Porto Alegre, RS, Brazil). An assessor monitored the pressure applied by the raters for two consecutive days using the same software, but the raters did not receive any visual feedback. The training was aimed at ensuring the velocity to apply pressure using a low-cost adapted algometer $(1 \mathrm{~kg} / \mathrm{s})$. The independently trained raters collected the middle deltoid muscle's pressure pain threshold of 20 participants (10 women; $22 \pm 2$ years; $63 \pm 13 \mathrm{~kg} ; 160 \pm 10 \mathrm{~cm} ; 23 \pm 4 \mathrm{~kg} / \mathrm{cm}^{2}$ ). The exclusion criteria for participants included: body mass index $>28 \mathrm{~kg} / \mathrm{cm}^{2}$, any self-reported health issues, alcohol consumption within five days prior to the assessments, shoulder pain, previous shoulder surgery, or any diagnosed shoulder or cervical impairment. The objectives of the study were explained to the subjects, who were notified of the benefits and potential risks involved before signing an informed consent form prior to participation. The Federal University of Juiz de Fora ethics committee for human investigation approved the procedures employed in the study (reference number: 02599418.9.0000.5147).

The pressure pain threshold was collected twice (3 days apart: days 1 and 2). To evaluate the intra- and inter-rater reliability, the following positioning was adopted: 1) the participant remained seated with the feet on the floor, 2) the hands rested on the thighs, and 3) the trunk was erect. The middle deltoid's site received progressive $1 \mathrm{~kg} / \mathrm{s}$ pressure controlled by a metronome until the participant experienced pain. ${ }^{8}$ An effort was made to standardize the anatomic locations of each session. The same rater was responsible for palpating and marking the pressure pain 
155 threshold site on each subject before any measurements, both on days 1 and 2 . The middle 156 deltoid's site was topographically determined in the middle of a horizontal line drawn between 157 the acromioclavicular joint and the deltoid muscle insertion. ${ }^{19}$ Three measurements were 158 performed for each site, with 10 to 15 seconds apart. The first measurement was discarded. ${ }^{20,21}$ 159 The participant lifted the opposite hand when the pressure pain threshold was achieved, that is, 160 when the applied pressed evoked pain. The examiner pressured the "tare" button to lock the 161 reading, immediately retracting the adapted pressure algometer. Then, the pressure pain 162 threshold reading was registered. ${ }^{22}$

163

164 Statistical analysis

165 The recorded peaks were then extracted. All trials were used for analysis, consisting of the 166 following: a total of 1) 60 measurements (validity analysis, force platform vs. adapted pressure 167 algometer) and 2) 80 measurements (reliability analysis). Data are presented as mean values and 168 standard deviations. The independent Student's t-test was used to compare differences between 169 measurements in the validation process. The intra- and inter-rater differences were compared using the mixed between- (rater 1 vs. rater 2) and within-subject analysis (moment and moment*rater) of variance with repeated measures. All data were reworked using Holm's post 172 hoc test to avoid multiple testing. Significance was set at $\mathrm{p}<0.05$. Intraclass correlation 173 coefficients $\left[\mathrm{ICC}_{(2,1)}\right]$ were calculated to compare the results between both types of equipment and raters. Poor reliability was indicated by values less than 0.5 , moderate reliability between 0.5 to 0.75 , good reliability between 0.75 and 0.9 , and excellent reliability greater than $0.90 .{ }^{23}$

176 Chronbach's $\alpha$ test was used to assess the expected correlation of both types of equipment 177 measuring the same construct. The standard error of measurement (SEM) was also calculated to 178 provide an estimate of measurement error. A linear regression was used to estimate the 179 coefficient of correlation $(r)$ and the adjusted coefficient of determination $\left(r^{2}\right)$. The magnitude of the correlation was qualitatively interpreted using the following thresholds: $<0.1$, trivial; $0.1-0.3$, small; $0.3-0.5$, moderate; $0.5-0.7$, large; $0.7-0.9$, very large; and $>0.9$, nearly perfect. ${ }^{24}$ The Bland-Altman method estimated the measurement bias, with lower and upper limits of agreement between results. Statistical analyses were performed using JAMOVI software (JAMOVI project, version $0.9,2018$ ). 
186 Results

187 Validity: Force platform vs. PA

188 No significant differences were observed in pressure trials (Table 1) between the adapted

189 pressure algometer $(405.63 \pm 235.34 \mathrm{kPa})$ and the force platform $(434.15 \pm 239.18 \mathrm{kPa} ; \mathrm{p}=0.25)$.

190 The $\operatorname{ICC}_{(2,1)}$ and Cronbach's $\alpha$ returned values of 0.98 and 0.99 , respectively. The SEM returned

191 a value of $0.005 \mathrm{kgf}$, and the linear regression showed statistically significant results $(\mathrm{r}=0.99$;

192 adjusted $\mathrm{r}^{2}=0.99 ; \mathrm{p}=0.001$ ). The Bland-Altman results showed high levels of agreement (Figure 193 2).

194

195

INSERT FIGURE 2 HERE

196

197

198

Intra- and inter-rater reliability

199

The pressure pain threshold from both raters showed very low variation over time (Rater 1: Day

199 $1=203 \pm 74 \mathrm{kPa}$, Day $2=206 \pm 71.6 \mathrm{kPa}$; Rater 2: Day $1=214 \pm 73.7 \mathrm{kPa}$, Day $2=215 \pm 69.6 \mathrm{kPa})$. The

200

201

202

203

204

205

206

207

208

209

210

211

212

213

214

215

intra-rater comparison showed no significant differences (Moment: $F=0.05 ; \mathrm{p}=0.83$ and Moment*Rater: $\mathrm{F}=0.01 ; \mathrm{p}=0.93$ ) (Table 1). The $\mathrm{ICC}_{(2,1)}$ and Cronbach's $\alpha$ analysis returned relevant values (Rater 1: $\operatorname{ICC}_{(2,1)}=0.76$, Cronbach's $\alpha=0.85$; Rater 2: $\operatorname{ICC}_{(2,1)}=0.73$, Cronbach's $\alpha=0.84$ ). The SEM values were low (Rater $1=0.02$, Rater $2=0.01$ ), and moderate values were also obtained in the linear regression analysis (Rater 1: $r=0.74$, adjusted $\mathrm{r}^{2}=0.52$; Rater 2: $\mathrm{r}=0.73$, adjusted $\mathrm{r}^{2}=0.50$ ). The Bland-Altman results showed high levels of agreement (Figure 3 ).

\section{INSERT TABLE 1 HERE}

The inter-rater reliability showed no differences among measurements $(\mathrm{F}=0.22 ; \mathrm{p}=0.64)$ (Table 1), with moderate results for reliability analysis (Day 1: $\operatorname{ICC}_{(2,1)}=0.56$, Cronbach's $\alpha=0.75$; Day 2: $\operatorname{ICC}_{(2,1)}=0.54$, Cronbach's $\alpha=0.71$ ). The SEM results showed very low values (Day 1: 0.04 kgf, Day 2: $0.02 \mathrm{kgf}$ ), and moderate values in the linear regression analysis (Day 1: $\mathrm{r}=0.60$, adjusted $\mathrm{r}^{2}=0.33$; Day 2: $\mathrm{r}=0.55$, adjusted $\mathrm{r}^{2}=0.26$ ). The Bland-Altman analysis showed acceptable levels of agreement (Figure 3).

\section{INSERT FIGURE 3 HERE}




\section{Discussion}

219 This study was designed to examine the instrumental validity and the intra- and inter-rater 220 reliability of an adapted low-cost pressure algometer. The results showed no significant

221 differences in the peak compressive force recorded from the adapted pressure algometer and the 222 force platform. A significant correlation was observed between the low-cost adapted algometer 223 and the force platform while measuring the same construct. These findings support the primary 224 hypothesis, which contends that a low-cost pressure algometer is both valid and reliable to be 225 considered as a standard equipment to assess the pressure pain threshold. Therefore, the tested device seems to be an acceptable alternative to expensive instruments. Previous studies showed acceptable levels of validity and reliability of other digital algometry systems. ${ }^{8,10}$ Kinser et al. ${ }^{8}$ tested the construct validity of a digital pressure algometer using the same protocol as our study by manually applying pressure on a force platform. The authors used ten sets of five applications to $80 \mathrm{~N}$ and one additional set of five applications to subsequent levels of progressive $10 \mathrm{~N}(20,30,40,50,60,70,80,90,100$, and $110 \mathrm{~N})$. The results showed

232 high correlation between the tested algometer and the force platform $(r=0.99)$ for both $80 \mathrm{~N}$ and incremental trials. Vaughan et al. ${ }^{12}$ also used the force platform as a reference instrument to test the validity of a digital pressure algometer. The authors applied 300 vertical pressures on the force platform with progressive pressure rates $(10,20,30,40$, and $50 \mathrm{kPa} / \mathrm{s})$. The result showed an excellent ICC range (0.90 to 0.99) for all comparisons. In general, all previous studies and the present work suggest the validity of the results obtained from a digital pressure algometer. These excellent results could be attributed to the strain gauge-based system used to acquire the signals. The conversion from analogic (load cell deformation) to electrical-digital signal (strain gauge) is very effective, even in very affordable systems. As the resistance varies in a sturdy element with

241 the applied force, the strain gauge converts the force (in this case, pressure) into a change in 242 electrical resistance that can be measured.

243 Several studies have evaluated the reliability of distinct pressure algometers as a tool to 244 distinguish healthy individuals from those with musculoskeletal disorders. Balaguier et al. ${ }^{25}$ 245 found high reliability between all three pressure pain threshold measures at sites in the lower 246 back. Walton et al. ${ }^{5}$ assessed the intra-rater and inter-rater reliability of an accessible digital 247 algometer in 60 healthy volunteers and 40 individuals with neck pain. The authors tested the 
248 upper fibers of the trapezius and tibialis anterior muscles. The intra-rater ICC results in both 249 groups ranged from 0.94 to 0.97 for the trapezius and tibialis anterior muscles. The inter-rater 250 ICC range (0.79-0.90) was lower than that of the intra-rater due to variations between observers. 251 However, both results were considered adequate. Waller et al. ${ }^{26}$ found high intra- and inter-rater 252 reliability ( $\mathrm{ICC}=0.81-0.99$; $\mathrm{ICC}=0.92-0.95$, respectively) using five research assistants. Each 253 assistant tested 20 pain-free subjects at the wrist, leg, cervical, and lumbar spine. The intra-rater

254 SEM ranged between 79 and $100 \mathrm{kPa}$. However, Van Wilgen et al. ${ }^{27}$ found lower values for 255 intra-rater reliability compared to inter-rater reliability of pressure algometry in healthy 256 volleyball athletes and those with patellar tendinopathy. The authors found high inter-rater 257 reliability $(\mathrm{ICC}=0.93)$, but only moderate intra-rater reliability $(\mathrm{ICC}=0.60)$ for pain pressure 258 threshold measurements. The authors argued that the lower intra-rater ICC values were probably 259 due to variance within the observer and also within the athletes, as the pain in patellar

260 261 262 263 264 265 266 267 268 269

270 271 272 273 274 275 tendinopathy varies over time. The diagnosis of fibromyalgia utilizes the pressure pain threshold as a key assessment to distinguish healthy individuals from those with fibromyalgia. ${ }^{28,29} \mathrm{Neck}$ pain, cranio-cervical headache, and temporomandibular disorders also include the pressure pain threshold as an important component for clinical reasoning about the level of severity, influencing the treatment direction. 5,9,30

However, those previous studies used commercial pressure algometers. For clinical and ambulatory settings, the high cost and the user's interface would be an issue to obtain fast objective pain measurements, requiring both training and experience for assessments. Brazilian physiotherapists have an average monthly salary of USD 500, according to the Occupational Brazilian Classification (https:/www.salario.com.br/profissao/fisioterapeuta-geral-cbo-223605/).

The adapted pressure algometer used in this study had a production cost of USD 10.00, while the standard digital equipment cost ranged from USD 600.00 to USD 1,000.00. The validation procedure enables use of the low-cost adapted pressure algometer for clinical assessments in a practice routine, which may directly impact primary and ambulatory care in low-income and developing countries, by adding an objective and inexpensive tool to assess the pressure pain threshold.

Some limitations of the present study must be addressed. The pressure pain threshold in body sites other than the deltoid muscle must be assessed to ensure the validity of the adapted pressure algometer on different sites. However, we hypothesize that they should not give any different 
279 results to direct assessment using the adapted pressure algometer, since the standard deviation 280 remained at very low values and the current results gave very good measures compared to the 281 force platform and additional good reliability. The instrumental validity of an equipment's 282 measurements also ensures unbiased assessments. ${ }^{31}$

283 Other studies have identified different factors to consider when evaluating the pressure pain 284 threshold, such as gender and obesity. ${ }^{32,33}$ A review of studies involving induced pain found a 285 consistent pattern of women exhibiting greater pain sensitivity and a reduction in pain inhibition compared to men. ${ }^{34}$ In addition, the characteristic of pain imposed is an important factor for these differences, since the type of pressure pain has one of the highest effect sizes in the pain report. $^{21,35}$ It is suggested that interactions between biological and psychosocial factors are responsible for these gender differences, but all studies indicate the need for additional research to elucidate the mechanisms that drive gender differences in pain responses. ${ }^{32,34,35}$ Some studies suggest that in areas with additional subcutaneous fat, pain thresholds for electrical or pressure stimuli increase and pain sensitivity decreases in obese individuals. ${ }^{33,36}$ A study has also shown biochemical changes in trigger points with higher levels of inflammatory mediators, catecholamines, and cytokines in obese individuals. ${ }^{37}$ Mechanical stretching of the skin in response to excess fat can lead to a decrease in the density of nociceptive fibers, and obesity is associated with the chemical inhibition of pain with an increase in $\beta$ endorphin and endogenous opioid peptide. ${ }^{33}$ The present study had a balanced cohort with regard to participant sex, and all participants were classified as normal according to their body mass index. However, the current sample was chosen only for reliability analysis. Pressure pain threshold as a clinical result is well established, but more studies should take into account sex and body mass index differences to avoid bias in experimental protocols. ${ }^{32}$ Pressure pain threshold was also positively but poorly correlated with high-density lipoprotein cholesterol. ${ }^{38}$ A high pressure pain threshold was also found among subjects with hyperglycemia and excessive alcohol consumption. ${ }^{38}$ In the present study, no blood assessment was performed to exclude those factors. However, the sample consisted of young adults, decreasing the chance of any important health issues. Additionally, exclusion criteria included previous excessive alcohol consumption.

307 Considering its portability, easy assembly, and lower cost, the currently tested device seems to be 308 a valid standard equipment for pressure pain threshold assessment. Therefore, the adapted pressure algometer is a valid device providing similar measurements compared to a force 
310 platform. The portability, cost-effectiveness, and friendly user system provide an effective way 311 to measure the pressure pain threshold.

312

\section{Conclusions}

314 The current hypothesis is that a low-cost pressure algometer is valid and reliable enough to be

315 considered as a standard equipment to assess the pressure pain threshold. The results showed that 316 the low-cost adapted pressure algometer is a valid tool compared to a force platform. The low317 cost adapted pressure algometer is also reliable for assessing the pressure pain threshold. Future 318 directions include evaluating the low-cost adapted pressure algometer in routine clinical 319 assessments for the systematic evaluation of pressure pain. Further studies should consider other 320 assessments, such as temporal summation and conditioned modulated pain, using a low-cost 321 adapted pressure algometer.

322

\section{3}

324 325

326

327

328

329

330

331

332

333

334

335

336

337 338

339

\section{Acknowledgements}

Special thanks to the UFJF-GV Department of Physical Therapy and to the Dean of International Relations - UFJF. We would like to thank Editage (www.editage.com) for English language editing.

\section{References}

1. Melia M, Schmidt M, Geissler B, et al. Measuring mechanical pain: The refinement and standardization of pressure pain threshold measurements. Behav Res Methods. 2015;47(1):216-227. doi:10.3758/s13428-014-0453-3

2. Imamura M, Chen J, Matsubayashi SR, et al. Cognitive performance is related to central sensitization and health-related quality of life in patients with chronic whiplash-associated disorders and fibromyalgia. Spine (Phila Pa 1976). 2013;38(24):2098-2107. doi:10.1097/01.brs.0000435027.50317.d7

3. Calixtre LB, Grüninger BL da S, Haik MN, Albuquerque-Sendín F, Oliveira AB. Effects of cervical mobilization and exercise on pain, movement and function in subjects with temporomandibular disorders: a single group pre-post test. J Appl Oral Sci. 
340

341

342

343

344

345

346

347

348

349

350

351

352

353

354

355

356

357

358

359

360

361

362

363

364

365

366

367

368

369

370

2016;24(3):188-197. doi:10.1590/1678-775720150240

4. Intelangelo L, Bordachar D, Barbosa AWC. Effects of scapular taping in young adults with shoulder pain and scapular dyskinesis. J Bodyw Mov Ther. 2016;20(3):525-532. doi:10.1016/j.jbmt.2015.11.014

5. Walton D, Macdermid J, Nielson W, Teasell R, Chiasson M, Brown L. Reliability, standard error, and minimum detectable change of clinical pressure pain threshold testing in people with and without acute neck pain. J Orthop Sports Phys Ther. 2011;41(9):644650. doi:10.2519/jospt.2011.3666

6. González-Fernández M, Ghosh N, Ellison T, McLeod JC, Pelletier CA, Williams K. Moving beyond the limitations of the visual analog scale for measuring pain: Novel use of the general labeled magnitude scale in a clinical setting. Am J Phys Med Rehabil. 2014;93(1):75-81. doi:10.1097/PHM.0b013e31829e76f7

7. Walton DM, Macdermid JC, Giorgianni AA, Mascarenhas JC, West SC, Zammit CA. Risk factors for persistent problems following acute whiplash injury: Update of a systematic review and meta-analysis. J Orthop Sports Phys Ther. 2013;43(2):31-43. doi:10.2519/jospt.2013.4507

8. Kinser AM, Sands WA, Stone MH. Reliability and validity of a pressure algometer. $J$ Strength Cond Res. 2009;23(1):312-314. doi:10.1519/JSC.0b013e31818f051c

9. Cunha CO, Pinto-Fiamengui LMS, Castro ACPC, Lauris JRP, Conti PCR. Determination of a pressure pain threshold cut-off value for the diagnosis of temporomandibular joint arthralgia. J Oral Rehabil. 2014;41(5):323-329. doi:10.1111/joor.12158

10. Durga P, Wudaru SR, Khambam SRKR, Chandra SJ, Ramachandran G. Validation of simple and inexpensive algometry using sphygmomanometer cuff and neuromuscular junction monitor with standardized laboratory algometer. J Anaesthesiol Clin Pharmacol. 2016;32(1):74-79. doi:10.4103/0970-9185.173390

11. Alburquerque-Sendín F, Madeleine P, Fernández-De-Las-Peñas C, Camargo PR, Salvini TF. Spotlight on topographical pressure pain sensitivity maps: A review. J Pain Res. 2018;11:215-225. doi:10.2147/JPR.S135769

12. Vaughan B, McLaughlin P, Gosling C. Validity of an electronic pressure algometer. Int J Osteopath Med. 2007;10(1):24-28. doi:10.1016/j.ijosm.2006.12.003

13. SJ K, H K, SK K, et al. Reliability and Validity of Modified Algometer in Abdominal 
$371 \quad$ Examination. Evid Based Complement Alternat Med. 2016;2016.

372 doi:10.1155/2016/3052954

373 14. O'Connor SM, Baweja HS, Goble DJ. Validating the BTrackS Balance Plate as a low cost

374

375 alternative for the measurement of sway-induced center of pressure. $J$ Biomech.

376 2016;49(16):4142-4145. doi:10.1016/j.jbiomech.2016.10.020

377

15. Hanafee JE, Radcliffe S V. Effect of High Pressure On a Strain Gauge Load Cell. Rev Sci

378 Instrum. 1967;38(3):328-331. doi:10.1063/1.1720698

16. Souza AC de, Alexandre NMC, Guirardello E de B. Propriedades psicométricas na 379 avaliação de instrumentos: avaliação da confiabilidade e da validade. Epidemiol e Serv 380 381 saude Rev do Sist Unico Saude do Bras. 2017;26(3):649-659. doi:10.5123/S167949742017000300022

382

17. Sullivan GM. A Primer on the Validity of Assessment Instruments. J Grad Med Educ.

383 2011;3(2):119-120. doi:10.4300/jgme-d-11-00075.1

384

18. Carlos-Vivas J, Martin-Martinez JP, Hernandez-Mocholi MA, Perez-Gomez J. Validation 385 of the iPhone app using the force platform to estimate vertical jump height. $J$ Sports Med 386 Phys Fitness. 2018. doi:10.23736/S0022-4707.16.06664-0

387

19. Ribeiro IL, Camargo PR, Alburquerque-Sendín F, Madeleine P, Fernández-de-las-Peñas 388 C, Salvini TF. Topographical pressure pain sensitivity maps of the shoulder region in 389 390

391

20. Ylinen J, Nykänen M, Kautiainen H, Häkkinen A. Evaluation of repeatability of pressure 392 algometry on the neck muscles for clinical use. Man Ther. 2007;12(2):192-197.

393 doi:10.1016/j.math.2006.06.010

21. Nussbaum EL, Downes L. Reliability of clinical pressure-pain algometric measurements 395 obtained on consecutive days. Phys Ther. 1998;78(2):160-169. doi:10.1093/ptj/78.2.160

396 397 398 399 400 401

22. Wytrążek M, Huber J, Lipiec J, Kulczyk A. Evaluation of palpation, pressure algometry, and electromyography for monitoring trigger points in young participants. $J$ Manipulative Physiol Ther. 2015;38(3):232-243. doi:10.1016/j.jmpt.2014.12.005

23. Koo TK, Li MY. A Guideline of Selecting and Reporting Intraclass Correlation Coefficients for Reliability Research. J Chiropr Med. 2016;15(2):155-163. doi:10.1016/j.jcm.2016.02.012 
402 24. Hopkins WG, Marshall SW, Batterham AM, Hanin J. Progressive statistics for studies in

403

404

405

406

407

408

409

410

411

412

413

414

415

416

417

418

419

420

421

422

423

424

425

426

427

428

429

430

431

432 sports medicine and exercise science. Med Sci Sports Exerc. 2009. doi:10.1249/MSS.0b013e31818cb278

25. Balaguier R, Madeleine $\mathrm{P}$, Vuillerme N. Intra-session absolute and relative reliability of pressure pain thresholds in the low back region of vine-workers: Ffect of the number of trials. BMC Musculoskelet Disord. 2016;17(1). doi:10.1186/s12891-016-1212-7

26. Waller R, Straker L, O’Sullivan P, Sterling M, Smith A. Reliability of pressure pain threshold testing in healthy pain free young adults. Scand J Pain. 2015;9:38-41.

doi:10.1016/j.sjpain.2015.05.004

27. Van Wilgen P, Van der Noord R, Zwerver J. Feasibility and reliability of pain pressure threshold measurements in patellar tendinopathy. J Sci Med Sport. 2011;14(6):477-481. doi:10.1016/j.jsams.2011.05.004

28. Gómez-Perretta C, Triñanes Y, González-Villar AJ, Carrillo-de-la-Peña MT. Evaluation of the accuracy of several symptoms and domains in distinguishing patients diagnosed with fibromyalgia from healthy controls. Clin Exp Rheumatol. 2016;34:14-25.

29. Cheatham SW, Kolber MJ, Mokha GM, Hanney WJ. Concurrent validation of a pressure pain threshold scale for individuals with myofascial pain syndrome and fibromyalgia. $J$ Man Manip Ther. 2018;26(1):25-35. doi:10.1080/10669817.2017.1349592

30. Castien RF, van der Wouden JC, De Hertogh W. Pressure pain thresholds over the craniocervical region in headache: a systematic review and meta-analysis. J Headache Pain. 2018;19(1). doi:10.1186/s10194-018-0833-7

31. Gadotti I, Vieira E, Dj M. Importance and clarification of measurement properties in rehabilitation. Rev bras fisioter. 2006;10(2):137-146. doi:10.1590/S141335552006000200002

32. Chesterton, LS Barlas, P Foster, NE Baxter, GD Wright C. Gender differences in pressure pain threshold in healthy humans. Int Assoc Study Pain. 2003;38:361-363. doi:10.1016/S0

33. Price RC, Asenjo JF, Christou N V., Backman SB, Schweinhardt P. The role of excess subcutaneous fat in pain and sensory sensitivity in obesity. Eur J Pain (United Kingdom). 2013;17(9):1316-1326. doi:10.1002/j.1532-2149.2013.00315.x

34. Bartley EJ, Fillingim RB. Sex differences in pain: A brief review of clinical and experimental findings. Br J Anaesth. 2013;111(1):52-58. doi:10.1093/bja/aet127 
433 35. Robinson ME, Riley JL, Brown FF, Gremillion H. Sex differences in response to 434 cutaneous anesthesia: A double blind randomized study. Pain. 1998;77(2):143-149. 435 doi:10.1016/S0304-3959(98)00088-8

436 36. Khimich S. Level of sensitivity of pain in patients with obesity. Acta Chir Hung. 437 1997;36(1-4):166-167.

438 37. Shah JP, Danoff J V., Desai MJ, et al. Biochemicals Associated With Pain and 439 Inflammation are Elevated in Sites Near to and Remote From Active Myofascial Trigger $440 \quad$ Points. Arch Phys Med Rehabil. 2008;89(1):16-23. doi:10.1016/j.apmr.2007.10.018

441 38. Zhang Y, Zhang S, Gao Y, et al. Factors associated with the pressure pain threshold in 442 healthy Chinese men. Pain Med (United States). 2013;14(9):1291-1300.

443 doi:10.1111/pme.12158

444

445

446 
Figure 1

Figure 1. Adapted pressure algometer - PA.

(1) Display; (2) On-Off button; (3) Tare button; (4) Unit selection button; (5) Adapted terminal. 


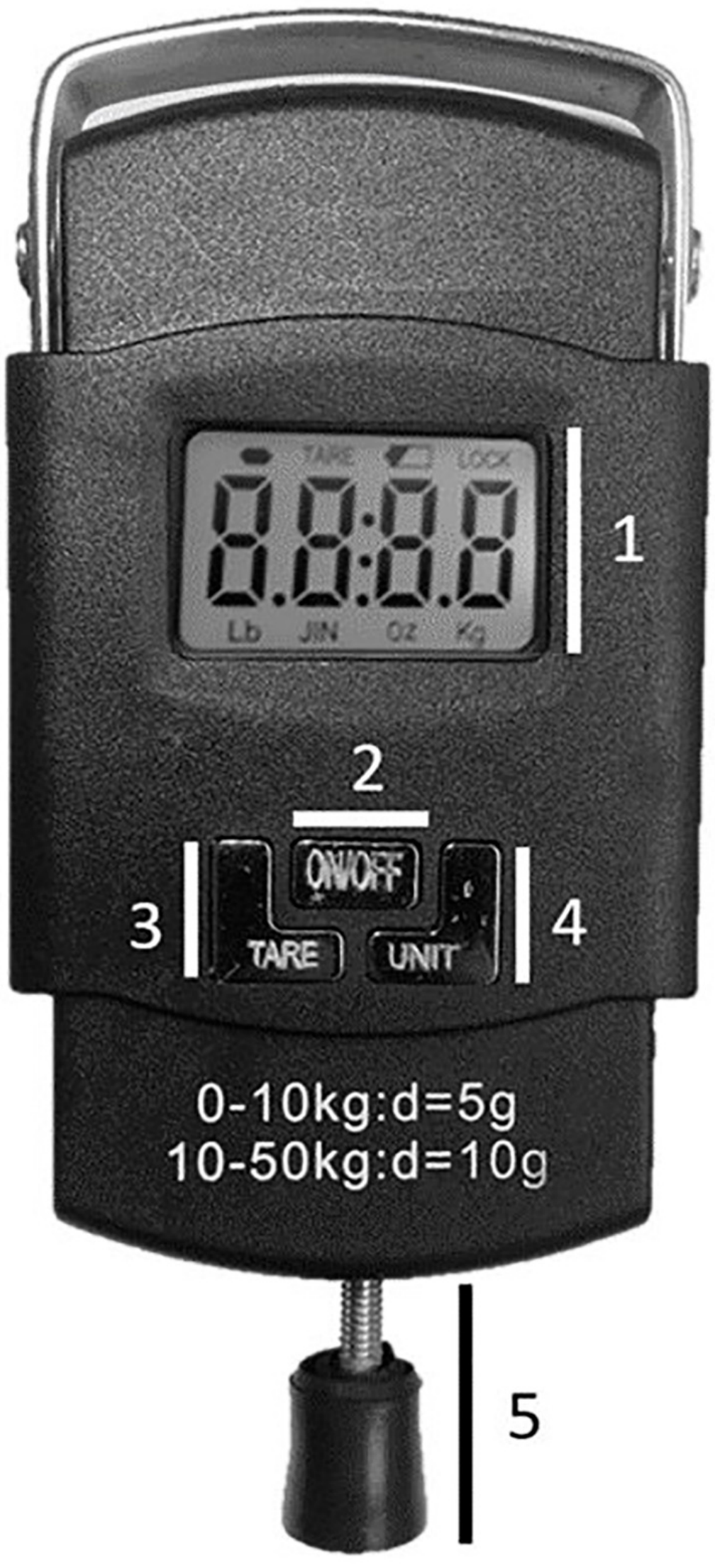


Figure 2

Figure 2. Bland-Altman plot: instrumental validity.

Bias $=-28.52(95 \%$ confidence interval $[\mathrm{Cl}]=-33.10$ to -23.9$)$; lower limit of agreement (LLA) $=-63.29(95 \% \mathrm{Cl}=-71.17$ to -55.4$)$; upper limit of agreement $($ ULA $)=6.25(95 \% \mathrm{Cl}=-1.62$ to 14.1).

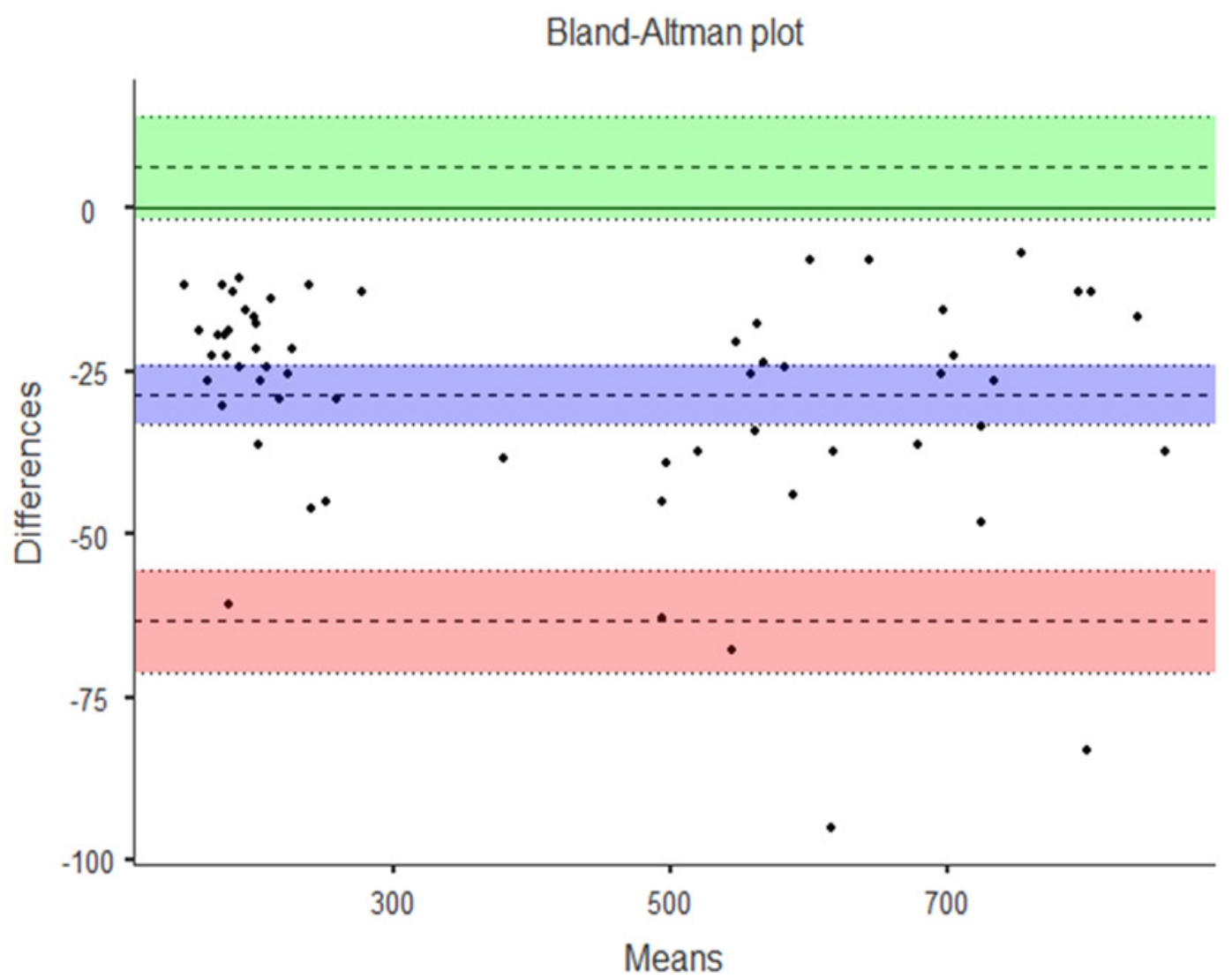


Figure 3

Figure 3. Bland-Altman plot: Intra-rater reliability.

Figure 3. Bland-Altman plot: Intra-rater reliability. A) Rater 1: Bias $=-2.55(95 \%$ confidence interval $[\mathrm{Cl}]=-24.3$ to 22.2$)$; lower limit of agreement $(\mathrm{LLA})=-106.38(95 \% \mathrm{Cl}=-149$ to -63.3); upper limit of agreement (ULA) $=101.28(95 \% \mathrm{Cl}=58.2$ to 144.4). 2). B) Rater 2: Bias $=-1.03(95 \% \mathrm{Cl}=-25.9$ to 23.9$) ; \mathrm{LLA}=-105.28(95 \% \mathrm{Cl}=-148.6$ to -62$) ; \mathrm{ULA}=103.22$ $(95 \% \mathrm{Cl}=59.9$ to 146 . Inter-rater reliability. C) Day 1: Bias $=-10.8(95 \% \mathrm{Cl}:-41.6$ to 20$)$; LLA $=-139.8(95 \% \mathrm{Cl}:-193.4$ to -86.3$) ; \mathrm{ULA}=118.2(95 \% \mathrm{Cl}: 64.7$ to 171.8$) ; \mathrm{D})$ Day 2: Bias = $-9.27(95 \% \mathrm{Cl}=-40.7$ to 22.1$) ; \mathrm{LLA}=-140.77(95 \% \mathrm{Cl}=-195.4$ to -86.2$) ; \mathrm{ULA}=122.23(95 \%$ $\mathrm{Cl}=67.6$ to 176.8$)$. 
A

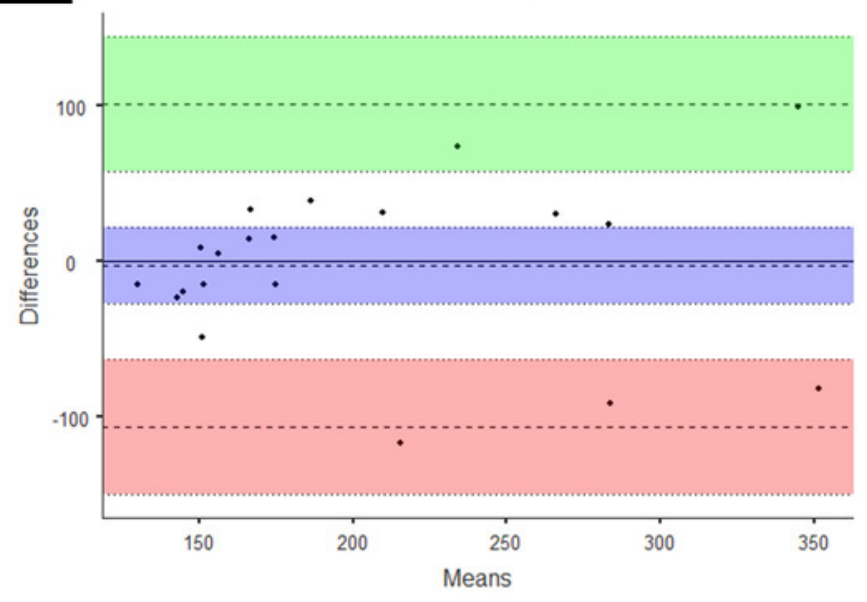

C

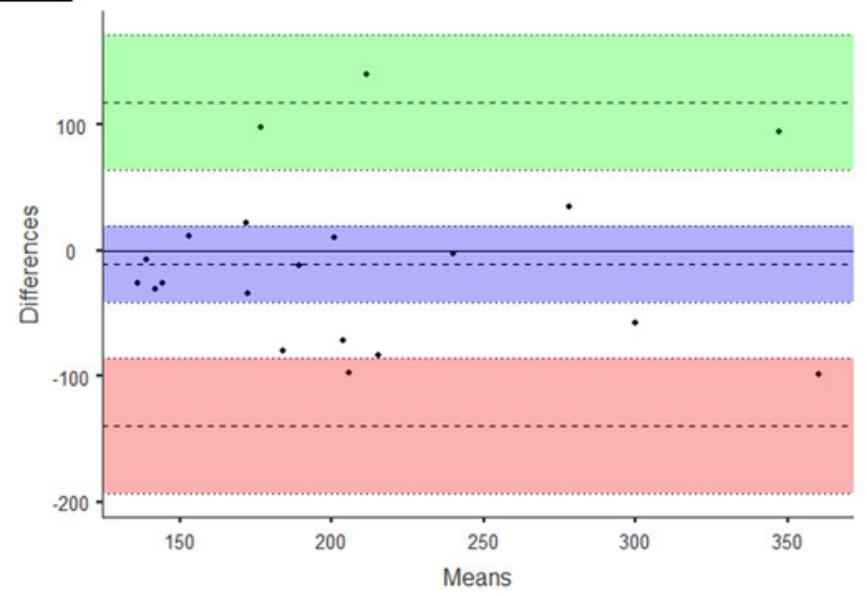

B $\quad$ Bland-Altman plot



D

Bland-Altman plot

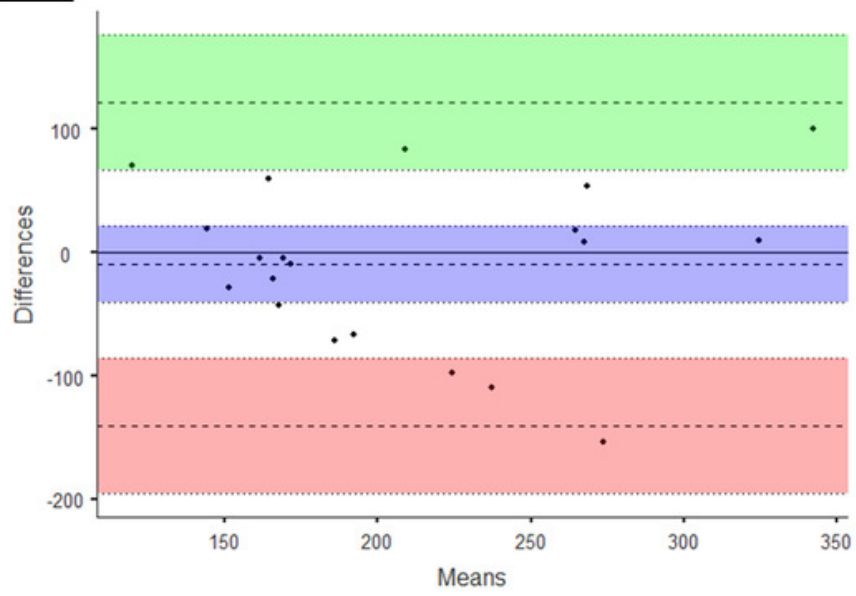




\section{Table $\mathbf{1}$ (on next page)}

Table 1: Validity, Intra- and Inter-rater reliability pairwise comparisons. 
1 Table 1:

2 Validity, Intra- and Inter-rater reliability pairwise comparisons.

3

\begin{tabular}{|c|c|c|c|c|c|}
\hline \multirow{2}{*}{ Type of Analysis } & \multirow{2}{*}{ Outcome } & \multirow{2}{*}{ p $_{\text {holm }}$} & \multirow{2}{*}{ Mean difference } & \multicolumn{2}{|c|}{ 95\% Confidence Interval } \\
\hline & & & & Lower & Upper \\
\hline \multirow[t]{2}{*}{ Validity } & $\begin{array}{c}\text { Adapted Pressure Algometer vs. Force } \\
\text { Platform }\end{array}$ & 0.512 & -28.5 & -114 & 57.3 \\
\hline & Rater 1 - Moment 1 vs. Moment 2 & 0.83 & -2.55 & -27.3 & 22.2 \\
\hline Intra-rater & Rater 2 - Moment 1 vs. Moment 2 & 0.93 & -1.03 & -25.9 & 23.9 \\
\hline \multirow{2}{*}{ Inter-rater } & Moment 1 - Rater 1 vs. Rater 2 & 0.65 & -10.79 & -58.1 & 36.5 \\
\hline & Moment 2 - Rater 1 vs. Rater 2 & 0.68 & -9.27 & -54.5 & 35.9 \\
\hline
\end{tabular}

4 